\title{
On Key Distribution in Secure Multicasting
}

\author{
Kuen-Pin Wu \\ kpw@orchid.ee.ntu.edu.tw
}

\author{
Shanq-Jang Ruan \\ stj@orchid.ee.ntu.edu.tw
}

\author{
Feipei Lai \\ flai@cc.ee.ntu.edu.tw
}

\author{
Chih-Kuang Tseng \\ tck@orchid.ee.ntu.edu.tw
}

\author{
Department of Electrical Engineering \\ and \\ Department of Computer Science and Information Engineering \\ National Taiwan University, Taipei 106, Taiwan
}

\begin{abstract}
Multicasting has been widely utilized for delivering messages from one sender to multiple recipients. Nowadays in some applications such as pay per view or videoconferencing systems, the messages delivered via multicasting should be available to authorized recipients only. Therefore, secure multicasting becomes an important design issue in distributed environment.

To achieve secure multicasting, all authorized recipients form a group and share a group key; messages should be encrypted by the key before they are multicasted. For the sake of security, we need a new group key if someone joins/leaves the group. Consequently, our goal is aimed at finding a way to distribute the new key securely and efficiently.

In this paper, we propose a new solution to the problem; we distribute new keys through a special function called secure filter. Compared with related work, our solution requires less system resources to change group members.
\end{abstract}

\section{Introduction}

Multicasting has been widely utilized for delivering messages from one sender to multiple recipients. For instance, in a pay-per-view system, the video server delivers a movie to all subscribers through multicasting. Another example is video-conferencing systems. In such systems, messages should be shown on the electronic white boards of all remote members, and the messages are sent via multicasting. Note that in the two examples, the multicasted material should be only available to authorized users. In other words, they should be protected from external intruders. Therefore, secure multicasting becomes an important design issue in distributed environment.
To achieve secure multicasting, all members in a multicast group share a group key; messages should be encrypted by the key before they are multicasted. On receiving the encrypted messages, group members can extract the plain messages using the group key, while others cannot. The security of such systems is therefore depends on the group keys.

Under this multicasting model, however, the system becomes insecure if someone joins/leaves the multicast group. When someone joins the group, he may access past multicasted messages using his newly obtained group key, but he is not an authorized recipient while these messages were sent. Similarly, after someone leaves the group, he may access succeeding encrypted messages using the group key he holds. He is not an authorized recipient of these messages, either. Consequently, the group key should be changed if someone joins/leaves the group. So, distributing new group keys will take place often in dynamic groups whose membership changes frequently. Our goal is hence aimed at finding a way to distribute new group keys securely and efficiently.

Many schemes have been proposed for secure multicasting. In some static key management protocols $[1,7,6]$, all members obtain an unchanged group key as they join the group. These schemes do not apply to dynamic groups. Chiou et al. [4] proposed a scheme called secure lock to broadcast securely, which is implemented using Chinese Remainder Theorem (CRT); only authorized members can extract the group session key from the secure lock. Performance issue becomes an important concern in this scheme, since CRT requires complex computation. Iolus [11] divides a large dynamic group into subgroups. Each subgroup requires an extra agent to deal with "key translation." Moreover, Iolus needs to encrypt the new group key for each member separately if a member exits, which takes a lot of computation time. The key management schemes for secure 
multicasting in $[3,5,13]$ require each of the $N$ members to store $\log (N)+1$ keys. As the group growing large, the system requires a great deal of keys; it is more difficult to manage so many keys and keep system secure. Cliques [9] provides a way to distribute group session keys in dynamic groups. However, it does not scale well to large groups. Molva et al. [12] proposed a scalable solution for dynamic multicast groups. Nevertheless, the scheme has to modify the structure of intermediate components of the multicast communication such as routers or proxies. Waldvogel et al. [10] proposed a scalable scheme for dynamic group such that each member holds fixed number of keys even if the group membership changes. In this scheme, if the system uses $k$ bits to represent a member ID, then each member holds $k+1$ keys. It is obviously that the system has up to $2^{k}$ members. The system has to be reorganized if the number of members exceeds $2^{k}$; on the other hand, if the number of members is far less than $2^{k}$, each member will hold relatively too many keys. However, a very low-overhead operation exists to shrink the key space as the system grows.

In this paper, we propose a new key distribution scheme for dynamic groups in secure multicasting; the scheme distributes the new group key through a special function called secure filter. ${ }^{1}$ In the proposed scheme, when a user joins/leaves the group, the central authority (CA) of the system first selects a new group key and encapsulates it into a secure filter $s f$, and then the CA multicasts $s f$. On receiving $s f$, the receiver $m_{j}$ first uses its own secret key $k_{j}$ to generate the input of $s f$, and then computes the value of $s f$. If $m_{j}$ is an authorized recipient, $s f$ returns the new group key to $m_{j}$, otherwise $s f$ returns an invalid value. The secure filter is the building block of our scheme, it greatly reduces the number of required encryption/decryption operations in the key distribution process.

The rest of the paper is organized as follows: Section 2 gives a formal definition of the key distribution problem in secure multicasting. Our scheme is then presented in Section 3. In the section, we first define the secure filter formally and describe its properties, then we use the secure filter to construct our solution. Security concern and performance evaluation of the solution are also given in this section. Section 4 concludes this paper.

\section{Key distribution in secure multicasting}

A secure multicasting environment consists of users and secure multicast groups. In our model, we assume that each user $m_{i}$ holds a private secret key $k_{i}$ to perform secure point-to-point communication. A secure multicast group $g_{j}$ is composed of more than one user. Moreover, $g_{j}$ is associated with a group key $g k_{j}$. Members of $g_{j}$ use $g k_{j}$ to per-

\footnotetext{
${ }^{1}$ Although not devoted to secure multicasting, the notion that users share secrets using a function can be found in [2].
}

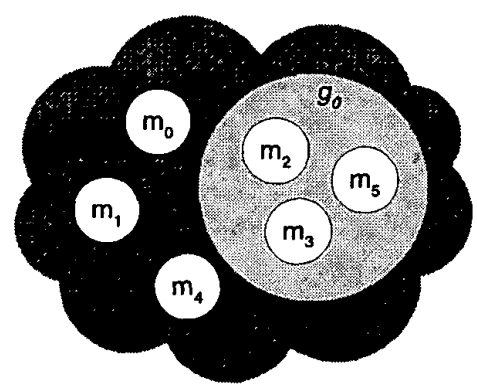

Figure 1. A secure multicasting environment.

form secure multicasting. Note that a user can be a member of more than one secure multicast group.

There is an example illustrated in Figure 1. In the figure, the secure multicasting environment has six users $m_{0}$, $m_{1}, \ldots, m_{5}$. A secure multicast group $g_{0}$ with three members $m_{2}, m_{3}$, and $m_{5}$ is also defined in the figure. Although not depicted in Figure 1, each user $m_{i}$ has a private secret key $k_{i}$ and the secure multicast group $g_{0}$ has a group key $g k_{0}$. Suppose that the member $m_{2}$ wishes to multicast a message, he first encrypts the message using $g k_{0}$, and then sends it to $m_{3}$ and $m_{5}$. On receiving the encrypted message from $m_{2}, m_{3}$ and $m_{5}$ use $g k_{0}$ to decrypt it.

Problems arise if the membership of $g_{0}$ changes. When a user joins $g_{0}$, he has to acquire $g k_{0}$ to encrypt/decrypt succeeding messages. However, it is possible that the user uses $g k_{0}$ to access past messages that are multicasted before he joins the group. This is not allowed because he is not an authorized recipient when the messages were sent.

Similarly, if a member leaves $g_{0}$, he is no longer authorized to access the succeeding multicasted messages. However, it is still possible that the member uses $g k_{0}$ to access them.

Therefore, group keys should be changed if some members join/leave the secure multicast groups. How to distribute new group keys securely is consequently an important design issue in dynamic groups whose membership changes frequently. Our goal is then primarily aimed at finding a way to distribute the new group key securely and efficiently. Moreover, when we distribute new group keys, we wish the required extra system resources as few as possible.

Our solution to the key distribution problem is presented in the next section. We define a special function as the building block of the solution. Discussions about the security and performance of the solution are also given in the section. 


\section{Our work}

In this section, we first define a special function called secure filter as the main building block of our solution to the key distribution problem. Then we describe our solution formally. Security concern and performance evaluation of our solution are also discussed. Finally we give a refinement of our solution.

\subsection{Secure filter}

A secure filter is a polynomial in the indeterminate $x$ over Galois field $\mathbf{G F}(p), p$ is a public large prime. For the set $C=\left\{k_{0}, k_{1}, \ldots, k_{t-1}\right\}$ and the number $g k$, where $g k$ and $k_{i}$, for $0 \leq i \leq t-1$, all belong to $\mathrm{Z}_{p}$, we can construct a polynomial $s f$ such that $s f(x)=\left(x-h\left(k_{0}\right)\right)(x-$ $\left.h\left(k_{1}\right)\right) \ldots\left(x-h\left(k_{t-1}\right)\right)+g k$. It is obviously that if $k_{j}$ belongs to $C, s f\left(h\left(k_{j}\right)\right) \equiv g k(\bmod p) . s f$ is then regarded as the secure filter on $g k$ with respect to $C$; where $h$ is a randomly chosen one-way hash function. For the sake of security, $s f$ is represented as $a_{t} x^{t}+a_{t-1} x^{t-1}+\ldots+a_{1} x+a_{0}$ in the system. Therefore, $g k$ cannot be extracted directly from $s f$.

In the following section, we use the secure filter to design our solution to the key distribution problem.

\subsection{The proposed solution}

Our solution to the problem is called KDSM (KDSM stands for Key Distribution in Secure Multicasting). In $\mathrm{KDSM}$, a central authority (CA) is to distribute/manage keys held by users. And further, all users share their private secret keys with the CA. Moreover, the CA holds all group keys. We assume that secret keys and group keys are chosen from $\mathbf{Z}_{p}$. Furthermore, mathematical operations are to be interpreted modulo $p$.

In the following two subsections, we describe how the CA works in KDSM when a user joins/leaves a secure multicast group.

Joining operation. There are two ways that the CA can distribute the new group key when a user joins a secure multicast group. The first way is the same as some proposed schemes in related work. When a user $m_{i}$ wishes to join the secure multicast group $g_{j}$, the CA first selects a new group key $g k_{j}^{\prime}$, and multicasts $E_{g k_{j}}\left(g k_{j}^{\prime}\right)^{2}$ to old members of $g_{j}$; finally, the CA sends $E_{k_{i}}\left(g k_{j}^{\prime}\right)$ to $m_{i}$ directly. The original members and $m_{i}$ can obtain the new group key $g k_{j}^{\prime}$ by decrypting the received messages using $g k_{j}$ and $k_{i}$ respectively. For an intruder, he cannot obtain $g k_{j}^{\prime}$ from $E_{g k_{j}}\left(g k j^{\prime}\right)$ or $E_{k_{i}}\left(g k j^{\prime}\right)$ without $g k_{j}$ and $k_{i}$; for

\footnotetext{
${ }^{2}$ The notation $E_{a}(b)$ means that the message $b$ is encrypted by key $a$.
}
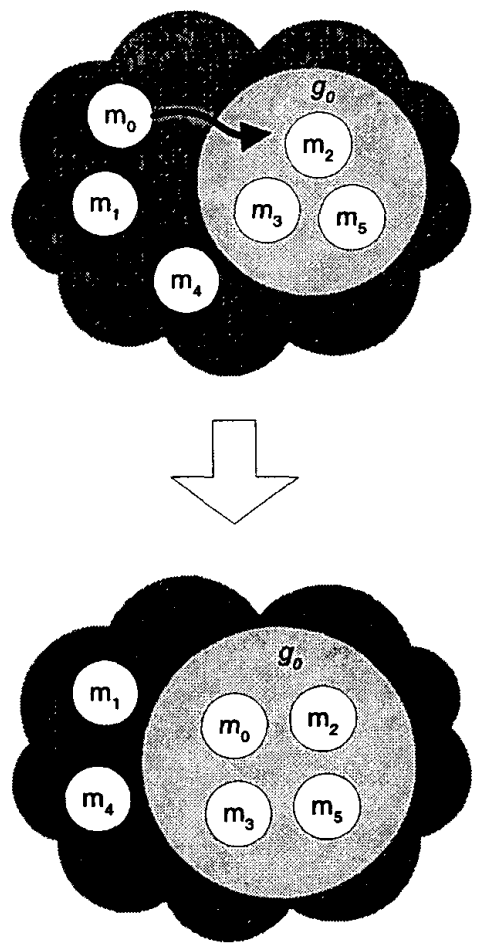

Figure 2. User $m_{0}$ joins the secure multicast group $g_{0}$.

$m_{i}$, he cannot access past encrypted messages without $g k_{j}$. Security requirement is therefore guaranteed.

The second way uses the secure filter. For a secure multicast group $g_{j}$, assume that $C_{j}$ is the set of private secret keys held by all members in $g_{j}$. If user $m_{i}$ wishes to join $g_{j}$, the CA selects a new group key $g k_{j}^{\prime}$ and a one-way hash function $h_{j}$, then the CA constructs a secure filter $s f_{j}$ on $g k_{j}^{\prime}$ with respect to $C_{j} \cup\left\{k_{i}\right\}$. Finally, the CA multicasts the message $\left(s f_{j}, h_{j}\right)$ to members of $g_{j}$ and $m_{i}$. On receiving the message, a user $m_{l}$ first figures out $h_{j}\left(k_{l}\right)$, then computes $s f_{j}\left(h_{j}\left(k_{l}\right)\right)$. If $m_{l}$ is a group member of $g_{j}$ or $m_{i}, k_{l}$ must be in $C_{j} \cup\left\{k_{i}\right\}$ and $s f_{j}$ will return the new group key $g k_{j}^{\prime}$ to $m_{l}$; otherwise $s f_{j}$ returns an invalid value. For an intruder, since his private secret key is not in $C_{j} \cup\left\{k_{i}\right\}$, he cannot acquire $g k_{j}^{\prime}$ form $s f_{j}$. Moreover, $m_{i}$ has no way to acquire $g k_{j}$ to access past encrypted messages in the scheme.

Example 1: Consider the scenario depicted in Figure 2. A new user $m_{0}$ joins the secure multicast group $g_{0}$. The CA first selects a new group key $g k_{0}^{\prime}$ and a one-way hash function $h_{0}$, then the CA constructs the secure filter $s f_{0}(x)=$ $\left(x-h_{0}\left(k_{0}\right)\right)\left(x-h_{0}\left(k_{2}\right)\right)\left(x-h_{0}\left(k_{3}\right)\right)\left(x-h_{0}\left(k_{5}\right)\right)+g k_{0}^{\prime}$. Finally, the CA sends $\left(s f_{0}, h_{0}\right)$ to $m_{0}, m_{2}, m_{3}$, and $m_{5}$. It is obviously that for $i \in\{0,2,3,5\}, m_{i}$ can acquire $g k_{0}^{\prime}$ by 

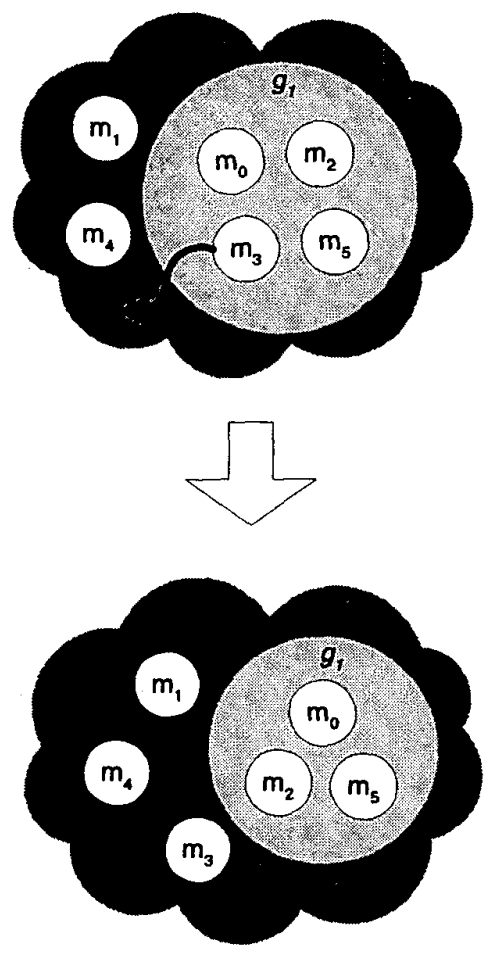

Figure 3. Member $m_{3}$ leaves the secure multicast group $g_{1}$.

computing $s f_{0}\left(h_{0}\left(k_{i}\right)\right)$.

Leaving operation. For the secure multicast group $g_{j}$, we assume that $C_{j}$ denotes the set of private secret keys held by all members in $g_{j}$. If some member $m_{i}$ of $g_{j}$ wishes to leave the group, the CA first selects a new group key $g k_{j}^{\prime}$ and a one-way hash function $h_{j}$, then the CA constructs a secure filter $s f_{j}$ on $g k_{j}^{\prime}$ with respect to $C_{j}-\left\{k_{i}\right\}$. Finally, the CA multicasts the message $\left(s f_{j}, h_{j}\right)$ to members of $g_{j}$ except $m_{i}$. On receiving the message, a user $m_{l}$ first figures out $h_{j}\left(k_{l}\right)$, then computes $s f_{j}\left(h_{j}\left(k_{l}\right)\right)$. If $m_{l}$ is a group member other than $m_{i}, k_{l}$ must be in $C_{j}-\left\{k_{i}\right\}$ and $s f_{j}$ will return the new group key $g k_{j}^{\prime}$ to $m_{l}$; otherwise $s f_{j}$ returns an invalid value. For an intruder, since his private secret key is not in $C_{j}-\left\{k_{i}\right\}$, he cannot acquire $g k_{j}^{\prime}$ form $s f_{j}$. Moreover, we guarantee that $m_{i}$ is no longer authorized to access further encrypted messages, because $k_{i}$ is not in $C_{j}-\left\{k_{i}\right\}$ and $m_{i}$ has no way to acquire $g k_{j}^{\prime}$.

Example 2: Consider the scenario depicted in Figure 3. Member $m_{3}$ leaves the secure multicast group $g_{1}$. The CA first selects a new group key $g k_{1}^{\prime}$ and a one-way hash function $h_{1}$, then CA constructs the secure filter $s f_{1}(x)=$ $\left(x-h_{1}\left(k_{0}\right)\right)\left(x-h_{1}\left(k_{2}\right)\right)\left(x-h_{1}\left(k_{5}\right)\right)+g k_{1}^{\prime}$. Finally, the
CA sends $\left(s f_{1}, h_{1}\right)$ to $m_{0}, m_{2}$, and $m_{5}$. It is obviously that for $i \in\{0,2,5\}, m_{i}$ can acquire $g k_{1}^{\prime}$ by computing $s f_{1}\left(h_{1}\left(k_{i}\right)\right)$.

\subsection{Security concern and Performance Evaluation of KDSM}

For security, it requires that unauthorized users cannot acquire the correct group keys to access data. Assume that an unauthorized intruder $u$ wishes to access the multicasted data in the secure multicast group $g$, he has to obtain the group key $g k$.

Since $u$ is not a member of $g$, he has only two possible ways to acquire $g k$. The first way is that $u$ extracts $g k$ directly from the intercepted secure filter $s f=$ $\left(x-h\left(k_{i 1}\right)\right)\left(x-h\left(k_{i 2}\right)\right) \ldots\left(x-h\left(k_{i j}\right)\right)+g k . u$ can only obtain $g k$ by extracting it from the constant term of $s f:(C+g k) \bmod p$, where $C=h\left(k_{i 1}\right) h\left(k_{i 2}\right) \ldots h\left(k_{i j}\right)$. Without knowing the numbers $h\left(k_{i 1}\right), h\left(k_{i 2}\right), \ldots$, and $h\left(k_{i j}\right)$, it is infeasible for $u$ to obtain $g k$, because $p$ is a large prime.

The second way is that $u$ first acquires some authorized member $m_{i n}$ 's private secret key $k_{i n}$, and then computers $s f\left(h\left(k_{i n}\right)\right)$ to obtain $g k$. Because $m_{i n}$ keeps $k_{i n}$ private, the only way that $u$ may obtain $k_{i n}$ is to extract it from the intercepted $s f$. To extract $k_{i n}$ from $s f, u$ has to extract $h\left(k_{i n}\right)$ first. Whether $u$ can get $h\left(k_{i n}\right)$ or not, it is infeasible that $u$ can extract $k_{i n}$ from $h\left(k_{i n}\right)$, because $h$ is a one-way hash function. Therefore, the security of KDSM is guaranteed.

Now we analyze the performance of KDSM from three aspects. We first consider the storage issue. For the secure multicast group $g_{j}$, unlike most related work, a member $u_{i}$ holds only a private secret key $k_{i}$ and a group key $g k_{j}$; other work usually requires more keys. Only the CA has to keep more objects in KDSM; the CA has to keep the private secret keys of all users and the group keys of all secure multicast groups.

For the computation load, when the CA distributes a new group key, only a few, even none, encryption/decryption operations are required in KDSM. Two primary operations may be performed by the $\mathrm{CA}$ or members: constructing secure filters and extracting new group keys from secure filters. To construct a secure filter, recall that the definition of secure filter $s f$ on $g k$ with respect to $S=$ $\left\{k_{0}, k_{1}, \ldots, k_{t-1}\right\}$ is $s f=\left(x-h\left(k_{0}\right)\right)\left(x-h\left(k_{1}\right)\right) \ldots(x-$ $\left.h\left(k_{t-1}\right)\right)+g k$. Transferring it into the form $a_{t} x^{t}+$ $a_{t-1} x^{t-1}+\ldots+a_{1} x+a_{0}$ requires $O\left(t^{2}\right)$ multiplications and $O\left(t^{2}\right)$ additions. To extract $g k$ from $s f$, suppose $s f$ is the polynomial over $\mathbf{G F}(p)$ of degree $t$, then it requires $t$ multiplications, $t$ additions, and a modular operation for an authorized member to extract the group key from $s f$ by applying Horner's rule [8]. Note that we have to compute the 
value of one-way hash functions in KDSM often, which can be done efficiently if the hash functions are well-selected.

Finally, for the message consideration, to distribute a new group key in KDSM, the CA only needs to multicast one message $(s f, h)$. This feature is better than most researches.

\subsection{Refinement}

Note that the degree of a secure filter is proportional to the size of the associated group. If the group grows large, constructing a secure filter requires a great deal of computation. To overcome this drawback, we can partition the group into subgroups, and the size of subgroups is bounded into a small range. Moreover, each subgroup is associated a subgroup key. When the membership of the group is altered, for the subgroups that do not involve the joining/leaving member, the CA first encrypts the new group key using their subgroup keys, and then multicasts the encrypted group key to them. The members of these subgroup can extract the new group key by their subgroup keys.

For the subgroup involving the joining/leaving member, the CA first constructs a secure filter for the subgroup, and then multicasts the secure filter to the members of the subgroup. Though extra keys and encryption/decryption operations are required in the modified scheme, we get a better performance to distribute a new group key if the system is large enough.

\section{Conclusion}

We proposed a solution KDSM to the key distribution problem in secure multicasting environment. To develop the solution, we suggest a special function called secure filter as the building block of KDSM. The function prevents unauthorized users from getting group keys, and then makes the key distribution process secure and efficient.

The KDSM ensures the system security; and further, it has low storage requirement, efficient objects generating/extracting operations and few message requirement. Note that the secure filters can be dropped directly after the new keys have been distributed.

\section{References}

[1] A. Ballardie. Scalable multicast key distribution. RFC 1949, May 1996.

[2] D. Bonch and M. Franklin. An efficient public key traitor tracing scheme. In Proc. Crypto 199, Lecture Notes in Computer Science, volume 1666, pages 338-353. SpringerVerlag, 1999.

[3] M. G. C. K. Wong and S. S. Lam. Secure group communication using key graphs. In Proc. ACM SIGCOMM '98, pages 68-79.
[4] G. H. Chiou and W. T. Chen. Secure broadcasting using the secure lock. IEEE Trans. Software. Eng., 15:929-934, 1989.

[5] E. J. H. D. M. Wallner and R. C. Agee. Key management for multicast: Issues and architectures. RFC 2627, June 1999.

[6] H. Harney and C. Muckenhirn. Group key management protocol (gkmp) architecture. RFC 2094, July 1997.

[7] H. Harney and C. Muckenhirn. Group key management protocol (gkmp) specification. RFC 2093, July 1997.

[8] D. E. Knuth. The Art of Computer Programming, Seminumerical Algorithms. Addison-Wesley, Reading, Mass., 1998.

[9] G. T. M. Steiner and M. Waidner. Cliques: A protocol suit for key agreement in dynamic groups. Technical report, IBM Zurich Res. Lab, Switzerland, Res. Rep. RZ 2984 No. 93030, Dec. 1997.

[10] D. S. N. W. M. Waldvogel, G. Caronni and B. Plattner. The versakey framework: Versatile group key management. IEEE J. Selected area in Commun., 17:1614-1631, Sept. 1999.

[11] S. Mittra. Iolus: A framework for scalable secure multicasting. In Proc. ACM SIGCOMM '97, pages 277-288.

[12] R. Molva and A. Pannetrat. Scalable multicast security in dynamic groups. In Proc. ACM CCS '99, pages 101-112.

[13] G. I. D. M. M. N. R. Canetti, J. Garay and B. Pinkas. Multicast security: A taxonomy and some efficient constructions. In Proc. INFOCOM '99, pages 708-716. 